\title{
ONTOLOGY-BASED APPROACH TO DATA EXCHANGES FOR ROBOT NAVIGATION ON CONSTRUCTION SITES
}

SUBMITTED: February 2021

REVISED: July 2021

PUBLISHED: July 2021

GUEST EDITORS: Kirti Ruikar, Ketan Kotecha, Sayali Sandbhor, Albert Thomas

DOI: $10.36680 /$ j.itcon.2021.029

\author{
Sina Karimi \\ Construction Engineering Department, École de Technologie Supérieure (ÉTS), Montréal, Québec, Canada; \\ sina.karimi.1@ens.etsmtl.ca \\ Ivanka Iordanova \\ Construction Engineering Department, École de Technologie Supérieure (ÉTS), Montréal, Québec, Canada; \\ ivanka.iordanova@etsmtl.ca \\ David St-Onge \\ Mechanical Engineering Department, École de Technologie Supérieure (ÉTS), Montréal, Québec, Canada; \\ david.st-onge@etsmtl.ca
}

SUMMARY: As the use of autonomous Unmanned Ground Vehicles (UGV) for automated data collection from construction projects increases, construction stakeholders have become aware of a problem with interdisciplinary semantic data sharing and exchanges between construction and robotic. Cross-domain data translation requires detailed specifications especially when it comes to semantic data translation. Building Information Modeling (BIM) and Geographic Information System (GIS) are the two digital building technologies used to capture and store semantic information for indoor structures and outdoor environments respectively. In the absence of a standard format for data exchanges between the construction and robotic domains, the tools of both industries have yet to be integrated into a coherent deployment infrastructure. In other words, the semantics of BIM-GIS cannot be automatically integrated by the robotic platforms currently being used. To enable semantic data transfer across domains, semantic web technology has been widely used in multi-disciplinary areas for interoperability. This paves the way to smarter, quicker and more precise robot navigation on construction sites. This paper develops a semantic web ontology integrating robot navigation and data collection to convey the meanings from BIM-GIS to the robot. The proposed Building Information Robotic System (BIRS) provides construction data that are semantically transferred to the robotic platform and can be used by the robot navigation software stack on construction sites. To meet this objective, first, knowledge representation between construction and robotic domains is bridged. Then, a semantic database integrated with the Robot Operating System (ROS) is developed, which can communicate with the robot and the navigation system to provide the robot with semantic building data at each step of data collection. Finally, the BIRS proposed system is validated through four case studies.

KEYWORDS: BIM, GIS, ROS, mobile robot, ontology, navigation, data exchange

REFERENCE: Sina Karimi, Ivanka Iordanova, David St-Onge (2021). Ontology-based approach to data exchanges for robot navigation on construction sites. Journal of Information Technology in Construction (ITcon), Special issue: 'Next Generation ICT - How distant is ubiquitous computing?', Vol. 26, pg. 546-565, DOI: 10.36680/j.itcon.2021.029

COPYRIGHT: (C) 2021 The author(s). This is an open access article distributed under the terms of the Creative Commons Attribution 4.0 International (https://creativecommons.org/licenses/by/4.0/), which permits unrestricted use, distribution, and reproduction in any medium, provided the original work is properly cited. 


\section{INTRODUCTION}

Manual data collection in the construction industry is inefficient due to its high cost, inaccuracy, and error-prone nature (Rebolj et al., 2017). The use of autonomous robots has shown great potential to achieve efficiency and high data collection precision (Ardiny et al., 2015). Among the different propositions, there is the implementation of new technologies leveraging cross-functional teams (Barbosa et al., 2017) and Scrum strategy deployment in various phases of construction (Streule et al., 2016). Studies have shown that one of the most important applications of mobile robots on construction sites is to monitor progress (Albeaino and Gheisari, 2021). As Albeaino et al. (2019) argue, an Unmanned Aerial Vehicle (UAV), also known as a drone, acquires construction data using commercial sensors such as laser scanners, cameras, radio frequency identification, and ultrasonic beacon systems. While UAV is mainly used for outdoor data acquisition, autonomous rovers mapping the environment, also known as Unmanned Ground Vehicle (UGV), complements the application of UAV for indoor environments where a Global Positioning System (GPS) is limited (Lin and Golparvar-Fard, 2020). Another approach adopted by Asadi et al. (2020) uses an integrated UAV-UGV system for construction data acquisition when UGV capabilities are limited due to the construction sites being cluttered with many obstacles. Similarly, the authors leveraged UAV to address UGV limitations to provide comprehensive and accurate data (Asadi et al., 2020). The fundamental requirement of automated data collection is for the robot to be able to (1) navigate autonomously in a dynamic environment and (2) acquire relevant and accurate data. Robot navigation mainly refers to the robot's ability to determine its position in a given environment, plan the path to the defined goal and move towards the goal's location (M. A. K. Niloy et al., 2021). With the enhanced capabilities of mobile robots in recent years, the interest to integrate high-level semantic information within robots is increasing: it is expected to ultimately make the robots easier to deploy (Crespo et al., 2020). Robots with semantic representation and recognition (association of location-semantic) of their environment are more intuitive to operate for non-experts, because they share conceptual understanding (Kostavelis and Gasteratos, 2017). One of the challenges to the implementation of semantic navigation is the association between high-level information and the geometry of the environment, such as discrete maps often referred to as occupancy grids. Occupancy grids are the environment's map with navigable space and obstacles through or around which the robot is autonomously navigating ("IEEE Standard for Robot Map Data Representation for Navigation," 2015). To achieve this, one needs to develop knowledge representation (Crespo et al., 2020), for which domain-specific ontologies are well suited (Gruber, 1995). Thus, this study aims to facilitate data exchange between construction and robotic domains by developing an ontology that supports the formal representation of the domains' knowledge. The resulting ontology then becomes a base structure for data extraction of BIM-GIS relevant to robot navigation. The current study is part of a larger research project whose focus is automated data collection and progress monitoring of construction projects.

BIM as a digital building model has various application; from design to maintenance state tracking (Doumbouya et al., 2017), smart city integration (Afsari et al., 2019), 4D simulation (Hatori et al., 2020), and clash detection (Savitri and Pramudya, 2020). In a similar way, GIS has enabled the Architecture, Engineering, Construction (AEC) industry to acquire geo-spatial data with regards to the surrounding site conditions and topographical information of a construction site (Karan and Irizarry, 2015). The integration of BIM and GIS grants new opportunities in construction projects such as finding the optimal location for tower cranes (Irizary and Karan, 2012), assessing the occupants' behavioral impact on energy consumption (Afkhamiaghda et al., 2019), assessing urban energy performance (Yamamura et al., 2017), helping with emergency response route (Tashakkori et al., 2015), merging indoor-outdoor combined route planning (Teo and Cho, 2016) and pre-construction planning (Karan and Irizarry, 2015). The integration of BIM-GIS data provides a holistic view of a digital built environment that includes both the facility and its surrounding environment at an urban. This representation can be used for robot navigation on construction sites to collect data on the existing conditions. However, to achieve this, the construction and robotic domains need to share a common semantic interoperability.

Semantic web technologies provide high-level data exchange among various domain knowledge representations, enabling interoperability by attaching decentralized data with semantics to different concepts (Kalfoglou, 2009). In an ontology, concepts, semantics and their relations with one another are defined through taxonomies (hierarchical structure of data) and relationships (Van Rees, 2003), thereby providing a machine-understandable structure in which concepts and knowledge are represented (El-Diraby et al., 2005). Ontologies can be categorized into three levels based on their components and level of detail, namely: top-level (or upper), domain, and application. Top-level ontologies formalize a generic ontology across all domains whereas domain ontologies 
provide knowledge representation that is formal, reusable, and shareable across a specific domain. Application ontologies aim to create focused ontologies for a given application (Karan Ebrahim P. et al., 2016).

The Institute of Electrical and Electronics Engineers published a standard (IEEE 1872-2015) ("IEEE Standard Ontologies for Robotics and Automation," 2015) for Robotics and Automation ontologies based on the Core Ontology for Robotics and Automation (CORA). The aim of this paper is to bridge IEEE 1872-2015 with construction-based knowledge. This standard provides a formal and shareable representation of the robotics and automation domain along with the definition of concepts and the relationships between concepts, attributions and constraints. The proposed ontology is a domain-type ontology that needs to be derived into specific implementations. Since some of the concepts discussed in the CORA are not thoroughly defined, CORAX (CORA's extension) was proposed to bridge these gaps ("IEEE Standard Ontologies for Robotics and Automation," 2015). The other standard leveraged for this study is the IEEE 1873-2015 ("IEEE Standard for Robot Map Data Representation for Navigation,” 2015). The Robot Map Data Representation for Navigation (MDR) standard focuses on the interoperability between robots, humans, and machines in terms of 2D metric and topological maps rather than 3D or semantic maps. Hence, the current study intends to bridge the semantics of BIM and GIS using some of the concepts in MDR to enrich map representation for human-robot data exchange.

Despite the number of studies using a mobile robot platform to acquire data on construction projects, semantic data translation between construction and robotic domains is still a problem requiring further research. Since there is no standard format to translate data between BIM-GIS and the Robot Operating System (ROS), the semantics of the IFC and CityGML are not understood by the robotic platform. This means the robot must "call" for the semantic information (Karan and Irizarry, 2015; Meschini et al., 2016). The current study's objective is to enable semantic interoperability between the BIM, GIS, and robot navigation system to translate semantic data from BIM and GIS to the ROS. The semantics of building elements can be provided to the ROS so that various information may be accessible to the robot when and where they are needed. The proposed bridging ontology is based on the extension of IEEE 1872-2015 and IEEE 1873-2015 standards. To reach this objective, a Building Information Robotic System (BIRS) is developed to convey semantic data to the ROS. The current study's contributions are:

1. An ontology bridging BIM-GIS data to IEEE 1872-2015 and IEEE 1873-2015 standards.

2. Cross-domain data structure enabling exchanges between construction and robotic domains.

3. A practical implementation of the proposed BIRS ontology and data structure that is deployed on an autonomous mobile robot navigating a construction site.

The first contribution of this paper is theoretical in that it provides a conceptual solution to bridge the knowledge representation of construction and robotic domains while the second and third are practical contributions providing the means for deploying the first contribution. The second contribution implements a data structure, which enables data exchanges between construction and robotic domains. The third contribution is the whole software structure for deployment of the proposed ontology in an autonomous robot integrated with navigation stack and robot's sensors.

Existing robot navigation solutions utilize different methods. Graph-based approaches such as A* and Dijkstra require the robot to have an a priori understanding of the environment with the obstacles' locations (Hassan et al., 2017) dividing the mobile robot environment into cells which finds the shortest distance between the start and the target connected through the cell graphs (Esan et al., 2020). Vector Field approach creates a new path in response to changes in the robot environment (Esan et al., 2020) by detecting and avoiding obstacles on the way to the target (Almasri et al., 2016). Genetic Algorithm adopts a problem-solving approach by addressing problems when they occur through several iterations (Wang et al., 2016). Aligning state-of-the-art navigation solutions, this paper provides waypoints for transiting from one space to another optimizing the existing navigation solutions.

This paper is structured as follows: Section 2 describes other scientific works relevant to this domain to provide an overview of what has been achieved and their contributions. Section 3 provides a detailed explanation of the methodology and how the proposed ontology is developed to facilitate data exchange. Section 4 presents how the ontology can be used as well as the results from four case studies. Finally, section 5 concludes the research carried out with the limitations of the proposed system as well as future research directions. 


\section{RELATED WORK}

Even though the growth of the World Wide Web has resulted in an incredible influx of information from heterogeneous data sources, users are able to navigate through the information easily enough. This demonstrates how powerful this semantic web is that it can be understood by both humans and machines (Berners-Lee and Hendler, 2001) for a domain-specific shared knowledge (Wang and Issa, 2020). The implementation of a semantic web requires the formal knowledge representation of the domains (ontologies) (Wang et al., 2019) in which their concepts and their interrelationship are explicitly described (Usmani et al., 2020). Ontology is defined in different ways in various fields of study. Guarino (1995) defined ontology as "an artefact constituted by a specific vocabulary used to describe a certain reality, along with a set of explicit assumptions related to the desired meaning of the vocabulary" in the context of Artificial Intelligence. Venugopal et al. (2015) defined ontology as a "knowledge representation mechanism" that provides an overview of a specific domain at an abstract level. Studer et al. (1998) defined ontology at a higher level as: "a formal, explicit specification of a shared conceptualization”. Pauwels et al. (2017), on the contrary, defined ontology and its applications at a lower level by "enabling data integration and complex search queries across several data sources." When it comes to developing an ontology, vocabulary does not suffice to convey the intended meaning. To achieve an efficient and functional ontology, other parameters must be taken into account, namely the concepts (terms that are mainly abstract and aligned with taxonomies), relationships (semantic connection between concepts), instances (an existing entity representing features of concepts) and axioms (defined valid rules across the domain) (González et al., 2020). The proven potentials of ontologies resulted in an increased interest in their use and the creation of novel forms of knowledge representation. Hence, the World Wide Web Consortium (W3C) developed the Resource Description Framework (RDF) to function as a "standard model for data interchange on the Web" (World Wide Web Consortium, 2014).

In the construction industry, the earliest studies on semantic web technologies aimed at achieving a higher level of efficiency for federated information by adopting an ontological approach to retrieve the "related concepts" to manage on-site problems (Elghamrawy and Boukamp, 2008). Another study used ontology to search and extract the construction data because the AEC data were numerous and somewhat hard to retrieve (Staub-French and Nepal, 2007). Sensing data integration for construction management through semantic web also received several earlier contributions. Elghamrawy and Boukamp (2010) developed an ontology in which Radio-frequency identification (RFID) technology is used to archive and retrieve construction document information. Liu et al. (2016) used ontology to estimate the costs of construction projects in China. Barbau et al. (2012) developed a plugin for a software tool supporting ontology development (i.e. Protégé) to translate the STEP's EXPRESS schema into the Web Ontology Language (OWL). Farias et al. (2015) proposed a framework to create an OWL ontology for COBie, a standard in BIM for Facility Management. González et al. (2020) described the development of an ontology to support indoor navigation that is based on IfcOWL ontology translating IFC schema into OWL. Venugopal et al. (2015) developed a model for data exchanges using IFC schema based on ontologies. Ontological approaches were also suggested for information exchanges in precast concrete (Venugopal et al., 2012). The literature shows that ontologies are nowadays common for information exchanges across the AEC industry but that they do not fully take advantage of established ontologies in other domains such as robotics. Therefore, we propose an ontology that bridges the gap between the construction and robotic domains.

Many other fields have leveraged ontologies to extend interoperability across domains. In the AEC industry, the information is by nature decentralized and fragmented (Atazadeh et al., 2017) which results in interoperability obstructions. Data from different sources come with different formats and follow different ontologies. The fact that each ontology is designed for different needs may obstruct interoperability within the AEC industry (Aziz et al., 2006). Hence, there has been a considerable number of attempts to provide an open source data schema for BIM interoperability such as BIMXML and COINS (Zhu et al., 2018). However, Industry Foundation Classes (IFC) is the primary open-source and EXPRESS-based data schema used across the AEC domain (Mignard and Nicolle, 2014). IFC schema is comprised of four layers, namely: resource layer, core layer, interoperability layer and domain/application layer (Terkaj and Viganò, 2017). IFC is a hierarchical data schema in which classes inherit the properties of upper layers (González et al., 2020). Many papers address the challenges of BIM - GIS integration and some of these adopt an ontological approach. More information on this and the requirements for using BIMGIS integration in robot navigation can be found in the review by Karimi and Iordanova (2021). 
There is also a number of ontology-based deployments in robotics outside of the construction domain. One of the first studies to use ontology is KnowRob (Knowledge processing for Robots) (Tenorth and Beetz, 2009). KnowRob ontology provides an open-source knowledge system for service robots managing uncertainties such as sensor noise. In the KnowRob ontology, the authors argue that controlling an autonomous robot requires several factors such as representing more fine-grained action. In another study (Beetz et al., 2018), the authors extended the KnowRob ontology to retrieve experimental knowledge ("narrative enabled episodic memory"). OpenEASE is a service, based on web knowledge using KnowRob to retrieve, store, supervise, and visualize the robot's knowledge (Beetz et al., 2015). KnowRob also uses RDF to represent the knowledge. RObot control for Skilled ExecuTion of Tasks in natural interaction with humans, based on Autonomy, cumulative knowledge and learning (ROSETTA) uses a set of ontologies to constitute a model in order for manufacturing robots to be adopted (Olivares-Alarcos et al., 2019). ROSETTA ontology is based on CORA ontology, which is in turn based on SUMO ontology. Although CORA uses the SUO-KIF language, ROSETTA is written in OWL. Persson et al. (2010) developed a "knowledge integration Framework" that establishes relationships between different segments of ROSETTA. OROSU (Ontology for Robotic Orthopedic Surgery) is yet another ontology, developed based on IEEE 1872-2015 Standard Ontology for Robotics and Automation, that uses OWL to retrieve information from the knowledge-based framework for surgical robotics (González et al., 2020). Diab et al. (2019) proposed a Perception and Manipulation Knowledge (PMK) ontology to represent and reason knowledge in task and motion planning. The PMK ontology is implemented in OWL based on CORA and SUMO (Olivares-Alarcos et al., 2019).

In the sub-domain of semantic knowledge for robot navigation, Kollar and Roy (2009) adopted an ontological approach to enable human-robot interaction, specific to the task of search and find. Galindo et al. (2005) proposed a multi-hierarchical approach for semantic navigation comprising of spatial and conceptual hierarchies. The former describes a conventional metric approach for building spaces and the latter incorporates semantic information of the environment. However, BIM semantics are still rarely used even though they could add more semantic information to robot navigation. While providing great contributions to semantic navigation, none of these studies considered incorporating BIM semantics to robot navigation during and/or after construction phases. And yet, several works address the use of BIM for robot navigation. Delbrügger et al. (2017) developed a framework for digital twin factories supporting human and robot indoor navigation. Ibrahim et al. (2017) studied the use of aerial robots to capture data from construction sites in an interactive way. Another study examined the use of BIM for localization of UAV in indoor environments taking advantage of AprilTags (Nahangi et al., 2018). Siemiątkowska et al. (2013) studied the use of BIM-based map representation in which the robot could localize semantically, using hierarchical path planning. Hamieh et al. (2017) developed a four-step BIM-based path planning strategy that uses hierarchical refinement of the number of paths. Palacz et al. (2019) proposed a graph-based approach for indoor navigation using BIM/IFC. TABLE 1 shows currently available ontologies found in the literature that use BIM information for indoor navigation. Required information in TABLE 1 refers to specific data of building elements which are needed to support ontology development.

TABLE 1. Currently available BIM-based ontologies supporting indoor navigation

\begin{tabular}{|l|l|l|l|}
\hline Application & $\begin{array}{l}\text { Built upon existing } \\
\text { ontologies }\end{array}$ & Required Information & Reference \\
\hline Indoor Navigation & - & $\begin{array}{l}\text { ColumnPart, BeamPart, SlabPart, DoorPart, } \\
\text { WindowPart, WallPart, MaterialType }\end{array}$ & (Isikdag et al., 2013) \\
\hline Information Reuse & ifcOWL & $\begin{array}{l}\text { IfcCartesianPoint, IfcPolyLoop, IfcPolyLine, } \\
\text { IfcMaterial, IfcWindow }\end{array}$ & (Pauwels et al., 2015) \\
\hline Indoor Positioning & - & $\begin{array}{l}\text { ID, Coordinate, Zone, Status, Level, Category, } \\
\text { Place_name }\end{array}$ & (S. Khruahong et al., 2017) \\
\hline $\begin{array}{l}\text { Occupants } \\
\text { Behaviour }\end{array}$ & $\begin{array}{l}\text { ifcOWL, SSN, W3C Time } \\
\text { Ontology, W3CGeo }\end{array}$ & $\begin{array}{l}\text { Occupant, Need, Driver, Action, BuildingState, } \\
\text { OccupantStateProperty, BuildingInfrastructure }\end{array}$ & (Chbeir et al., 2018) \\
\hline Indoor Routing & $\begin{array}{l}\text { GIS/OWL, IndoorGML } \\
\text { OWL }\end{array}$ & $\begin{array}{l}\text { IfcDoor, IfcSpace, IfcOpeningElement, Instnce ID, } \\
\text { GUID, OverallHeight, OverallWidth }\end{array}$ & (Wang and Issa, 2020) \\
\hline Indoor Navigation & ifcOWL & $\begin{array}{l}\text { IfcBuilding, IfcBuildingStorey, IfcDoor, IfcSite, } \\
\text { IfcSlab, IfcStair, IfcWall, IfcWindow, } \\
\text { IfcTypeObject, IfcPresentationLayerAssignement, } \\
\text { IfcLocalPlacement, IfcGlobalPosition, } \\
\text { IfcAxis2Placement3D }\end{array}$ & (González et al., 2020) \\
\hline
\end{tabular}


Despite their great contributions, none of the aforementioned works studied the automatic translation of semantic data from BIM to the ROS. There have also been several research attempts in which the authors used GIS for robot navigation (Mirats-Tur et al., 2009; Qiangrong Yang et al., 2015; Yan et al., 2013). However, the integration of BIM and the additional information that can be used for robot navigation has yet to be thoroughly studied.

\section{RESEARCH METHODOLOGY}

The current study proposes a novel approach in which BIM and GIS data are used in robot navigation during/after the construction phase. The research methodology is comprised of two steps: (1) develop the Building Information Robotic System (BIRS) Ontology to bridge IFC and CityGML with the IEEE 1872-2015 and IEEE 1873-2015, and (2) enable cross-domain interoperability through BIRS Data Exchange. Figure 1 illustrates the pipeline and establishes the practical implementation of the robotic system using BIM and GIS for navigation.

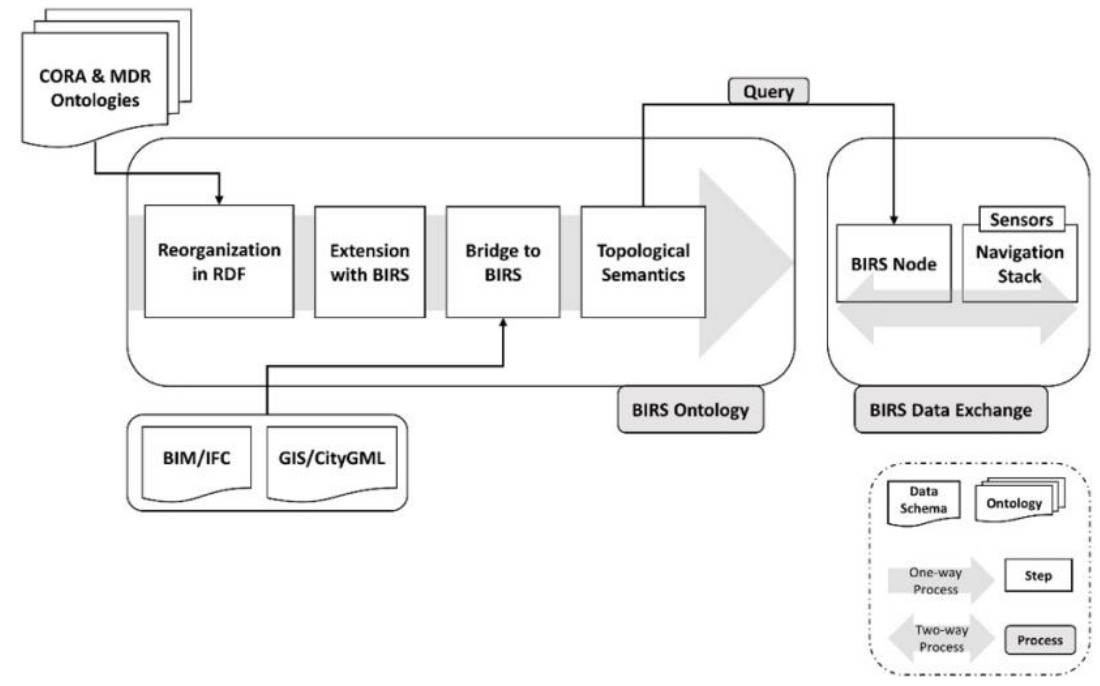

FIG. 1. Proposed pipeline for the implementation of the Building Information Robotic System (BIRS), supporting development of BIRS Ontology and BIRS Data Exchange.

As illustrated in FIG. 1, the first step (BIRS Ontology) creates an ontology bridge connecting IFC and CityGML data to CORA and MDR. The second step (BIRS Data Exchange) reasons the BIRS ontology to create a BIRS node in ROS in order to translate the semantic data from the ontology to the robotic system. Both steps presented in the pipeline are explained in detail in the following sections.

\subsection{Building Information Robotic System (BIRS) Ontology}

The purpose of this section is to develop an ontology that supports the bridging of BIM and GIS to CORA and MDR. To achieve this, the existing IEEE standards need to be reorganized (the ontology classes and axioms remain intact) to facilitate the knowledge translation from engineering to application ontologies. CORA and MDR are expressed in RDF so as to be compatible with the development of median level classes. The combination of RDF and XML would enable users to exchange data among various applications (Karan and Irizarry, 2015). Since the resulting entities should comply with IEEE 1872-2015 and IEEE 1873-2015 standards, the intermediate level classes are developed to be integrated with the corresponding concepts from IFC and CityGML.

In order to extend CORA and MDR in our work, a short review of the underlying concepts is required. CORA extends the Suggested Upper Merged Ontology (SUMO), which is a top-level ontology (Prestes et al., 2013). SUMO supports the definition of high-level knowledge concepts in the world. The highest SUMO class is Entity, which has two further sub-classes; Physical and Abstract. The former describes the entities in 3D space and the latter represents concepts that do not have spatial shape features. In other words, Abstract contains anything which does not fall into Physical (Niles and Pease, 2001). Physical is a disjoint partition of Object and Process. Object describes existent objects in 3D space with no temporal effect on the space while Process follows a "perdurantist" approach that adds temporal effects and considers 4D orientation (Fiorini et al., 2015). Abstract, on the other hand, is defined as not being a subclass of Object. It has the following sub-classes: Quantity, Attribute, SetOrClass, Relation, and Proposition. Figure 2 illustrates the SUMO taxonomies from which CORA is developed. 


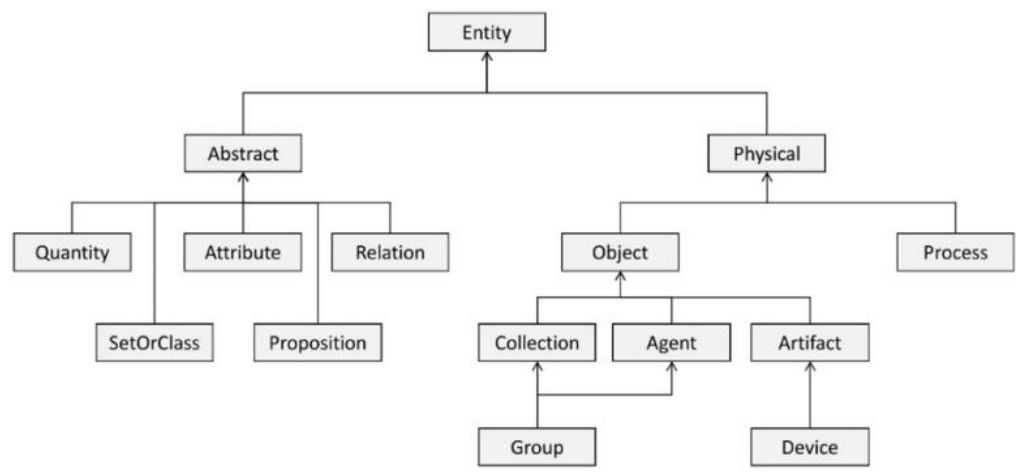

FIG. 2. Basic SUMO taxonomies [Source: ("IEEE Standard for Robot Map Data Representation for Navigation," 2015)]

IEEE 1872-2015 argues that "Concepts and relations associated with design, interaction, and environment" are too general to be incorporated in CORA and are not effectively covered by SUMO ("IEEE Standard Ontologies for Robotics and Automation," 2015). To bridge this gap, Fiorini et al. (2015) defined a new class Physical Environment as a three-dimensional environment which contains region and other artifacts existing in 3-D space dependent on the landmark. CORAX incorporates PhysicalEnvironment as a subclass of Object under Physical which is located in 3 dimensions and consist of one Region in minimum ("IEEE Standard Ontologies for Robotics and Automation," 2015). IEEE 1872-2015 also defines Design as a subclass of proposition, which has an idealization relationship between design and artifact. In SUMO ontology, content bearing physicals represents propositions such as a descriptive sentence, a graph, etc. No restriction is applied for ContentBearingPhysicals to represent an idea. Therefore, we integrate the MDR as the subclass of CORA:ContentBearingObject since MDR aims to facilitate data interoperability between robots. IEEE 1873-2015 standard ("IEEE Standard for Robot Map Data Representation for Navigation," 2015) subdivides maps into Metric and Topological, in which Metric maps are the disjoint entity of the Continuous Metric Maps and Discrete Metric Maps representing the physical layout of the environment and the objects within the robot's physical environment. Continuous metric maps are comprised of the geometric elements while Discrete metric maps utilize bitmap illustrations of the environment under which OccupancyGridMaps are categorized. OccupancyGridMaps are the most widely used maps in Simultaneous Localization and Mapping (SLAM) and in robot navigation, they are generally considered as Discrete Metric Maps. Topological maps are generally represented by sets of nodes and edges (Choi et al., 2011), which facilitates path planning tasks while running an algorithm on the created graph ("IEEE Standard for Robot Map Data Representation for Navigation," 2015). Figure 3 illustrates the overall integral graph in which all the ontologies come together and shape an integral ontology taxonomy along with the proposed BIRS entities.

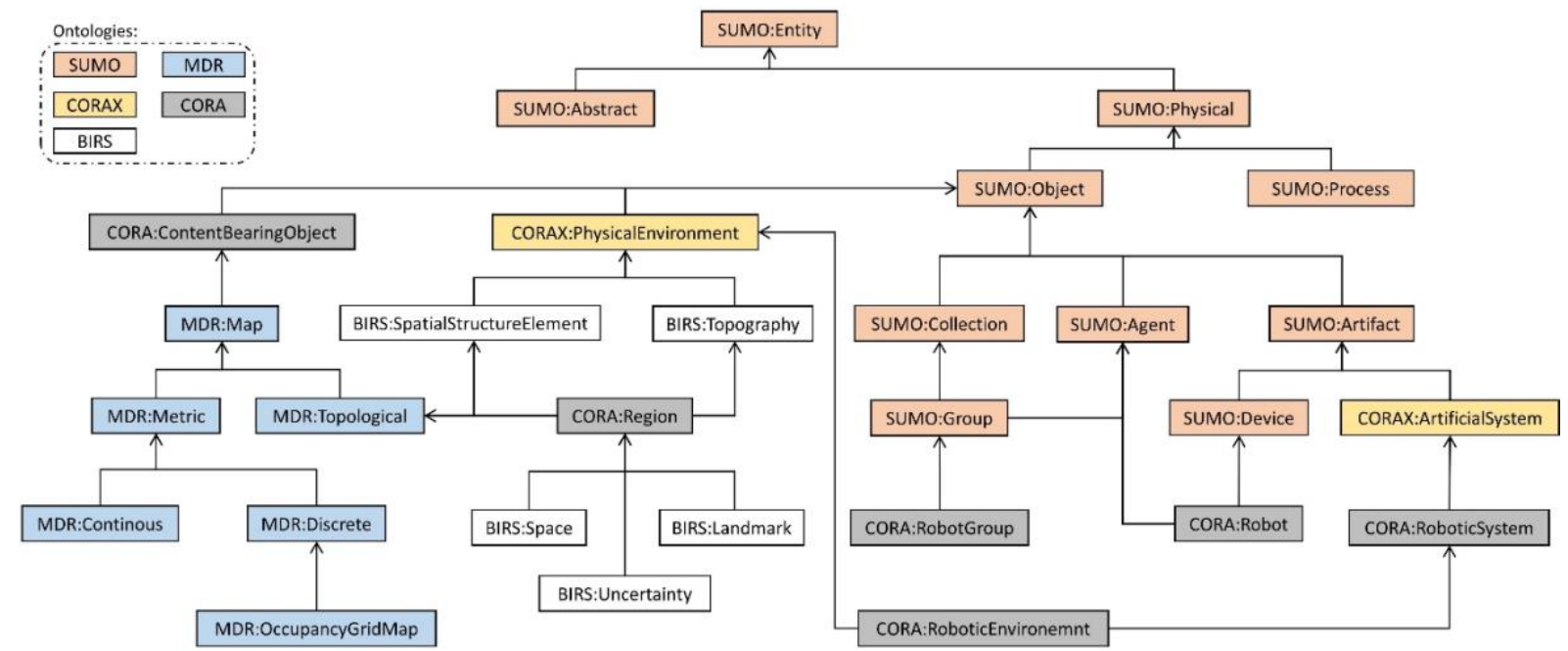

FIG. 3. Integration of CORA and MDR ontologies with Building Information Robotic System (BIRS) Ontology indicating sub-class relationships (:SubClassOf) [Sources: ("IEEE Standard for Robot Map Data Representation for Navigation," 2015; “IEEE Standard Ontologies for Robotics and Automation, " 2015)] 
We extend an intermediate level between CORAX:PhysicalEnvironment and CORA:Region to differentiate between indoor and outdoor environments. This is required by the digital representation of the built environment that correlates the building components and their semantic attributes with the topographic and existing conditions information of the construction site (Karan and Irizarry, 2015). Therefore, SpatialStructureElement and Topography can be the median level that define Region more effectively. We follow the extension of CORA and MDR by including Landmark, Space and Uncertainty as the sub-classes of Region bridging the construction physical environment comprised of BIM and GIS. TABLE 2 defines the new concepts provided in BIRS.

TABLE 2. Definition of BIRS concepts.

\begin{tabular}{|c|c|c|}
\hline Concept & Definition & Reference \\
\hline Landmark & $\begin{array}{l}\text { "A feature that is used for localization of a mobile robot. It is a feature } \\
\text { whose pose in a coordinate system can possibly be measured } \\
\text { (localized) by the robot's sensors with respect to a given map." }\end{array}$ & $\begin{array}{l}\text { ("IEEE Standard for Robot Map Data } \\
\text { Representation for Navigation," 2015) }\end{array}$ \\
\hline Space & $\begin{array}{l}\text { "Represents an area or volume bounded actually or theoretically. } \\
\text { Spaces are areas or volumes that provide for certain functions within a } \\
\text { building." }\end{array}$ & (buildingSMART, 2013) \\
\hline Uncertainty & $\begin{array}{l}\text { Dynamics of the construction site that cannot be considered as } \\
\text { landmarks and which have temporal effects on construction job-site. }\end{array}$ & - \\
\hline
\end{tabular}

Landmarks are the physical building elements that the robot can use for localization. IfcBuildingElement is the entity that defines the properties of the building elements. Semantics of classes and properties are defined through axioms in the proposed BIRS ontology. The SubClassOf axiom is a demonstration of the defined relationship between the higher and lower entities (Karan Ebrahim P. et al., 2016). For instance, since the IfcWall is a subclass of IfcBuildingElement, IfcWall inherits all the properties of IfcBuildingElement. It is also applicable to all IFC classes due to the fact that IFC is a hierarchical data schema. In BIRS, the building elements extracted as the subclass of IfcBuildingElement are IfcWall, IfcCurtainWall, IfcColumn, IfcDoor, IfcRailing, and IfcStair. Figure 4 illustrates the relationships of BuildingElement with a higher entity (landmark) and the properties associated with it in IFC schema. There are several essential properties in IfcBuildingElement required to translate a building layout into a ROS-compatible format. The shape and location of the landmark can be derived through ObjectPlacement and Material properties. IfcProduct is the super-type of the IfcBuildingElement through which the local placement is defined. The coordinates of the geometric representation of BuildingElement are defined through LocalPlacement. Using the landmark's material properties would contribute to robot navigation as well, but it would have to be accessible through a pipeline other than layout. Using the aforementioned IFC classes in the BIRS ontology, a BIM-based occupancy grid map is within reach. This map can be extracted in two ways. One is to use tools such as ifcConverter to export the map from IFC files to .svg, then to a ROS-compatible format such as .png, and the second method would use the BIM design authoring tools such as Autodesk® Revit and Graphisoft巴 ArchiCAD to export the map in .png format.

From the extracted data, a topological map with each space considered as a node is expected as the output. Figure 3 illustrates the relationships between space, topological map and CORA:Region. Hence, a space is a node that is bounded using the IfcRelSpaceBoundary relationship, which provides boundaries for building elements. As illustrated in FIG. 4, IfcRelSpaceBoundary provides relationships with landmarks and nodes used to generate the topological map containing the semantic information of the spaces and landmarks for the purpose of robot navigation (implemented in Python). The use of IFC semantics for robot navigation is twofold. It contains the information of the landmark material in case the robot gets into a space for which the laser provides a point cloud it is unable to segment. As well, the information of the nodes that represent space names would enable semantic navigation. Applying the ontology outdoors with knowledge of obstacles helps the robot to avoid collision. 


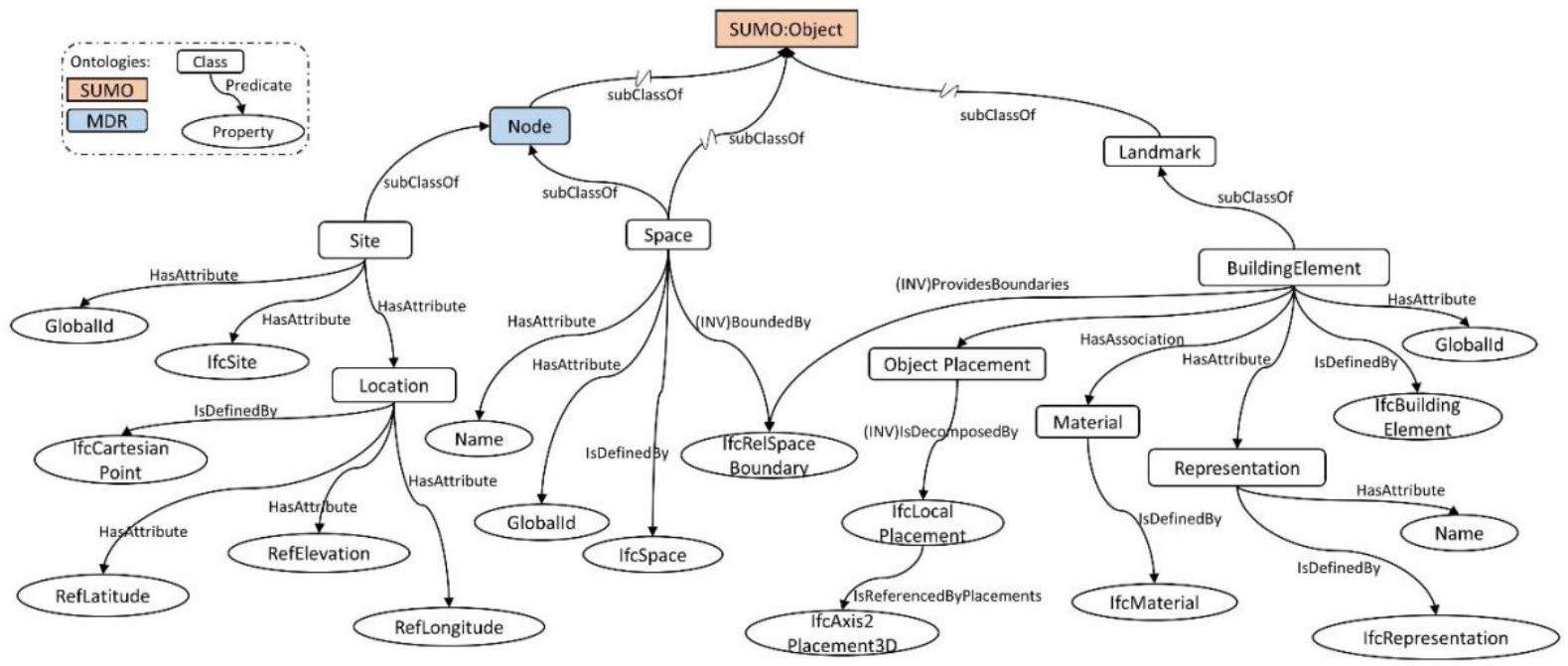

FIG. 4. Partial indication of IFC classes relationships in BIRS ontology.

To make it possible for the robot to gather information about the building's surroundings, existing and natural artifacts are integrated and considered as obstacles, namely: existing buildings, water surfaces and vegetation. Figure 5 illustrates the relationships between CityGML and the BIRS ontology. The most important properties of outdoor artifacts are their location. The geo-location properties of the landmarks are translated to BIM. Then, the integrated data are translated to the robot local positioning system to support obstacle avoidance. The procedure on how the GIS information is transferred to BIM is beyond the scope of the current study. More information on this can be found in (Karan Ebrahim P. et al., 2014).

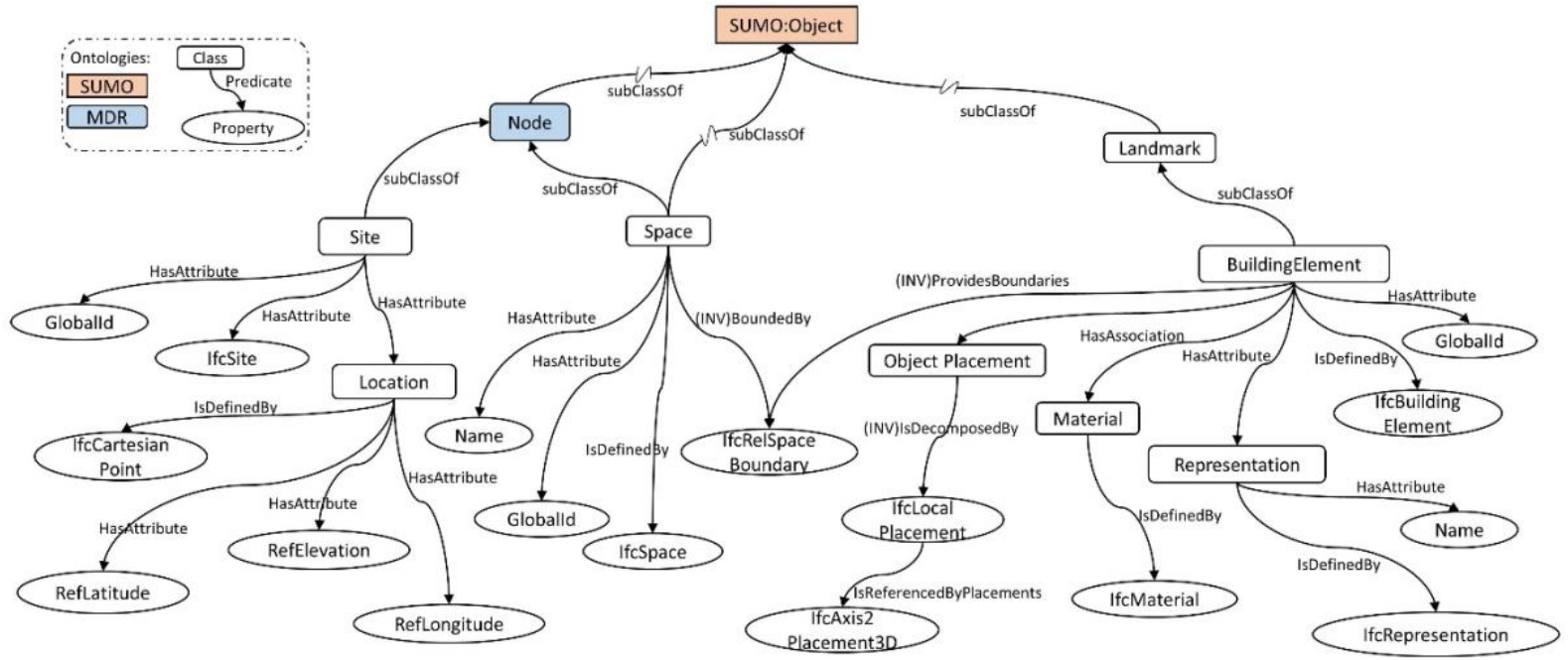

FIG. 5. Partial indication of CityGML classes relationships in BIRS ontology.

In the expected topological map, each pair of nodes are connected by an edge and since the edges are one-way relationships, the resulting graph is directional. Extracted data from BIM, the edges are the transition between spaces (nodes), which is done through the doors (edges). IfcDoor is the SubClass Of IfcBuildingElement containing height and width, which are defined through IfcDoor properties. Figure 6 illustrates the door relationships with regards to the landmark and the edge. Furthermore, IfcDoor entity stores the information of door opening direction through y-axis of ObjectPlacement. BIRS uses IfcDoor information to create the edges. The center of the door in IfcDoor is defined by IfcLocalPlacement, which inherits from its super-type IfcProduct. As illustrated in FIG. 6, a door is also a landmark which the robot can use to localize itself. If the spaces are not connected through a wall opening, the coordinates of wall opening are extracted through IfcLocalPlacement of IfcOpeningElement. 


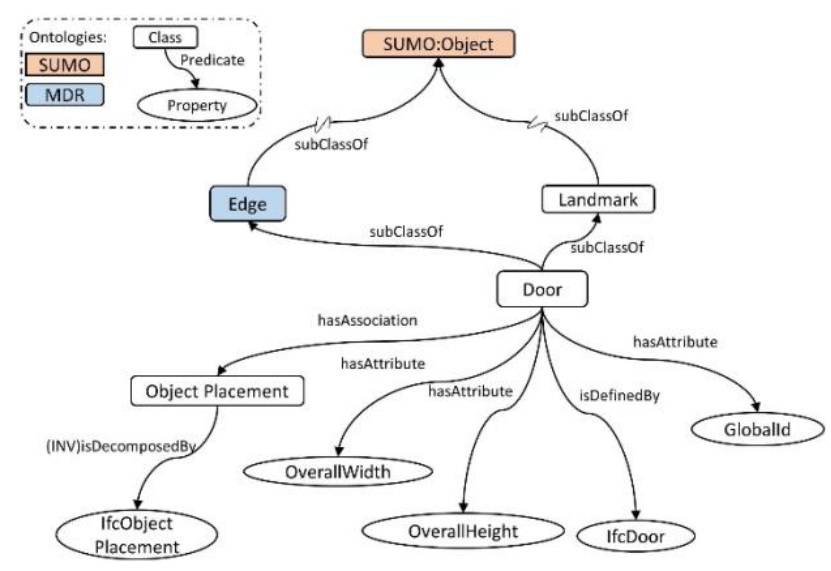

FIG. 6. IfcDoor relationships in BIRS ontology.

\subsection{Building Information Robotic System (BIRS) Data Exchange}

As illustrated in FIG.1, to achieve cross-domain data exchange using the BIRS ontology, the IFC file is imported in Autodesk $®$ Revit 2020 and processed with a Dynamo script (visual programming tool for Autodesk ${ }^{\circledR}$ Revit). The information needed to create a semantic topological map is retrieved using the IFC parameters defined by the BIRS ontology. The corresponding information is then exported as an XML database containing semantic information for the creation of the topological map. The semantic topological map was then created with the nodes and edges in a Python script. Since the data need to be accessible to ROS, this Python query script (ROS node) parses the BIRS information in the XML database. The semantic information from BIRS is not directly understood by the robot since the information is represented as strings with meaning in the ontology. Hence, the node includes dictionaries translating the complex information to machine-friendly scales. Apart from the rooms' names, all the information in the graph's nodes and edges follow the same translation process. Any other node in the ROS environment can then subscribe to retrieve semantic information. For example, when the robot enters a room that has a glass wall that is hard to detect by its sensors, the BIRS node provides the robot with enough information about the room so it can rely on the BIRS layout (occupancy grid). The BIRS node can quickly find the room where the robot stands from the room boundaries exported automatically using the low-level information of the navigation system.

Due to the nature of the construction projects, there is a high number of activities and machinery that temporarily operate during the construction phase and are not fully represented in BIM. Hence, those entities that cannot be addressed with BIM are categorized under Uncertainty in BIRS and are addressed with the low-level navigation stack and real-time sensors. The low-level navigation system leverages sensors on the UGV and state-of-the-art algorithms to enable the robot autonomous navigation on construction sites. High-level information is provided to the robot from BIM-GIS data, which gives the robot an a priori map (as discussed in section 3), as well as waypoints for navigation, localized building elements material and the information of nodes and edges in the topological map. All the sensing modalities (e.g. laser scanner, odometry, depth camera and inertial measurement unit) used for navigation, path planning and obstacle avoidance are subject to various noise, such as process noise, model noise and electric noise, generating Uncertainty. Detail on how low-level navigation operates in the presence of Uncertainty is beyond the scope of this paper.

\section{CASE STUDY}

Many use cases can potentially benefit from BIM-GIS integration into the robot navigation stack. The selected use cases aim to exemplify the application of BIM and GIS to help the robot understand the spatial structure and topographical environment in an autonomous navigation stack on construction sites. The context of the case is pavilion D $\left(2922.67 \mathrm{~m}^{2}\right)$ of École de technologie supérieure (ÉTS), which is covered with very light vegetation and surrounded by streets on two sides (southwest and southeast) and by buildings on the other sides. In FIG. 7, a topological graph database is created for the second floor of ÉTS' pavilion D with BIM/IFC information using the BIRS ontology. Each node includes a set of data identified and extracted using the BIRS ontological approach. BIRS information is then used to create a second map supported by ROS, the a priori metric map (occupancy grid). The following four use cases are practical deployments tested with a Clearpath Jackal mobile robot. 


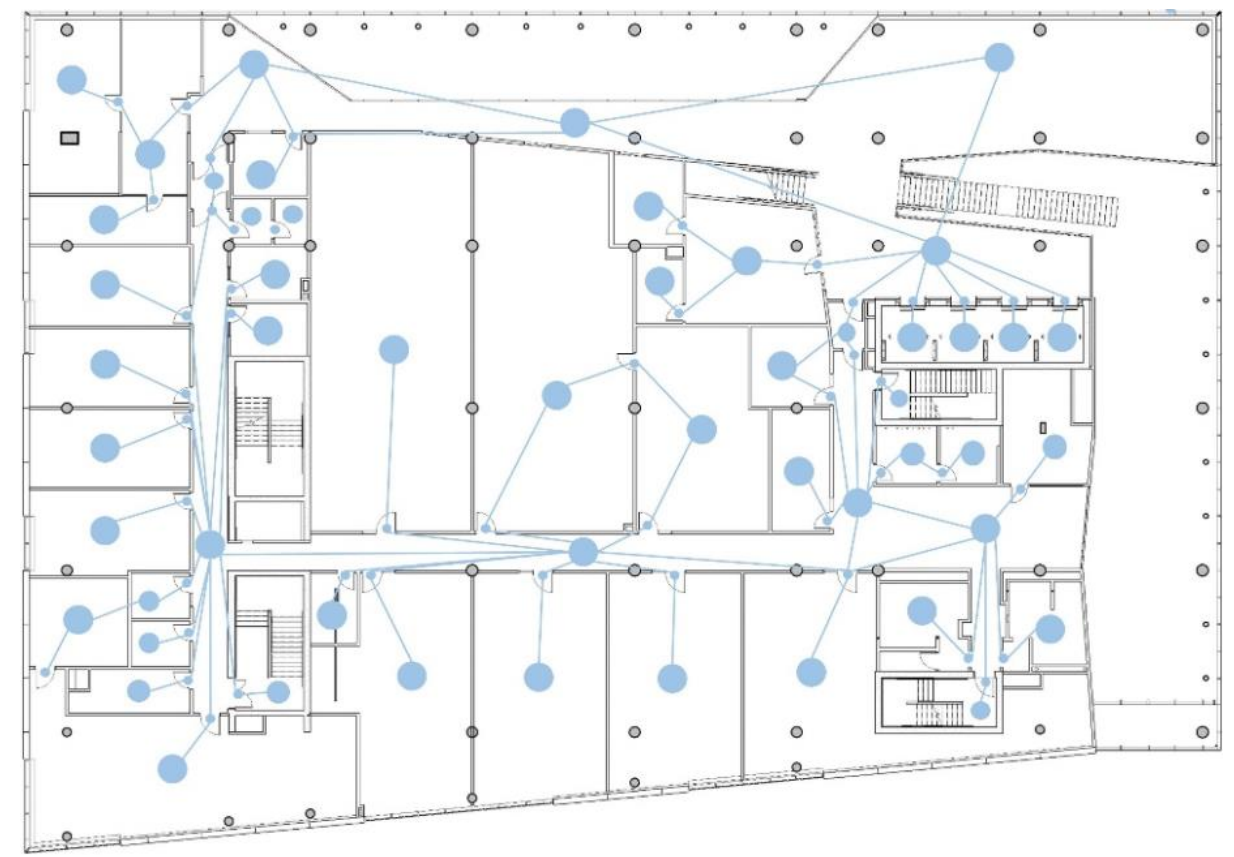

FIG. 7. Topological Map representation of ÉTS Pavilion-D.

\subsection{Semantic Indoor Navigation}

In this use case, the robot starts navigation and data collection from "CORRIDOR OUEST 2019" (west hallway) and is instructed to reach "W.C. HOMMES 2002" (men's bathroom) in the building. The destination, in the eastern section of the building, needs to be scanned while the starting point of the robot is in the western section. The coordinates of the desired room (center of the room) are provided by the topological map to the robot in order to reach the destination. The query script provides the destination coordinates in the BIRS node and the low-level navigation system subscribes to the node and fetches the required information. As illustrated in FIG. 8, the path to the destination is long with multiple rooms along the way, namely, "VESTIBULE 2043", "HALL 2044", "VESTIBULE 2042", "CORRIDOR EST 2007", and "ESPACE CLLABORATIF 2004". On its way to the destination node, the robot enters "HALL 2044", which has a large and high glass wall, invisible to the robot's sensor. Figure 8 illustrates the map created by the SLAM algorithm, which uses laser scanner for navigation.
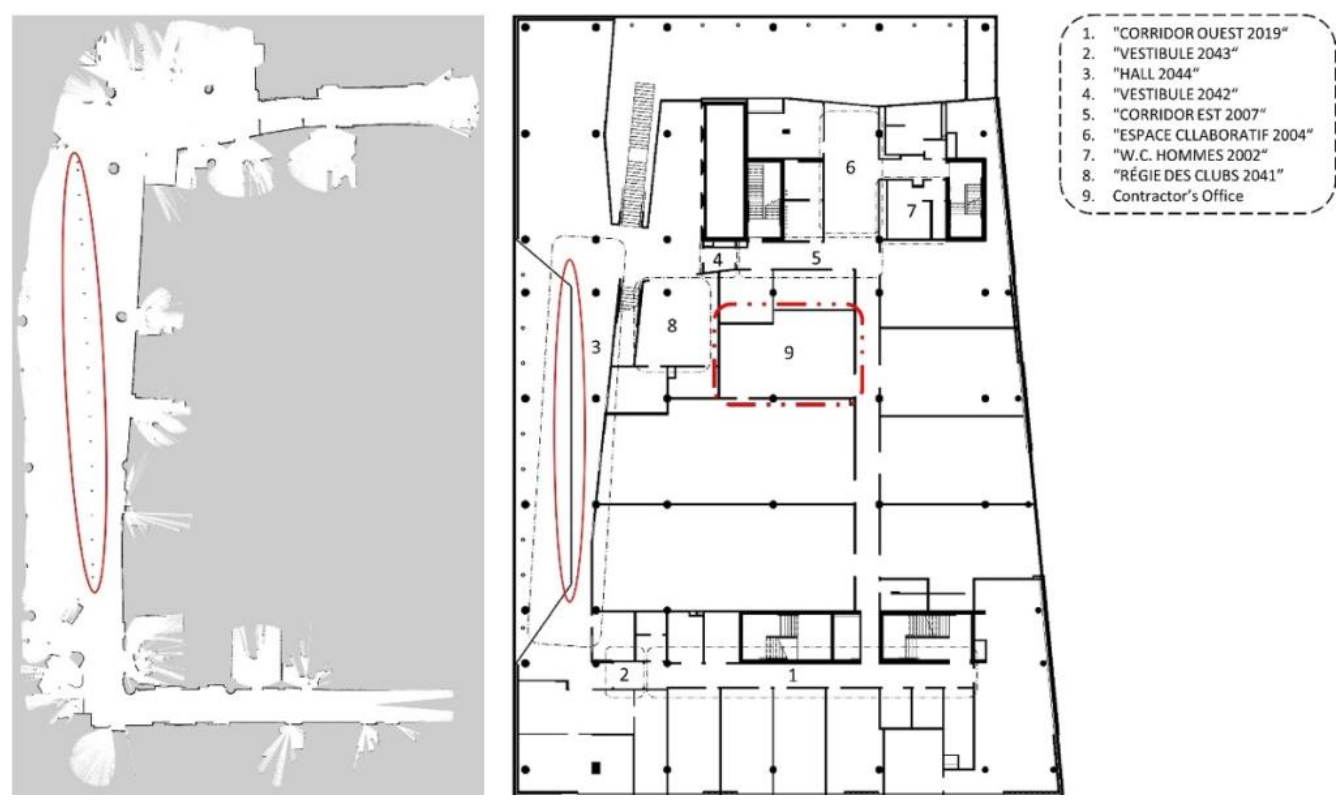

FIG. 8. Map generated by SLAM algorithm (left) and map created from BIM (right). 

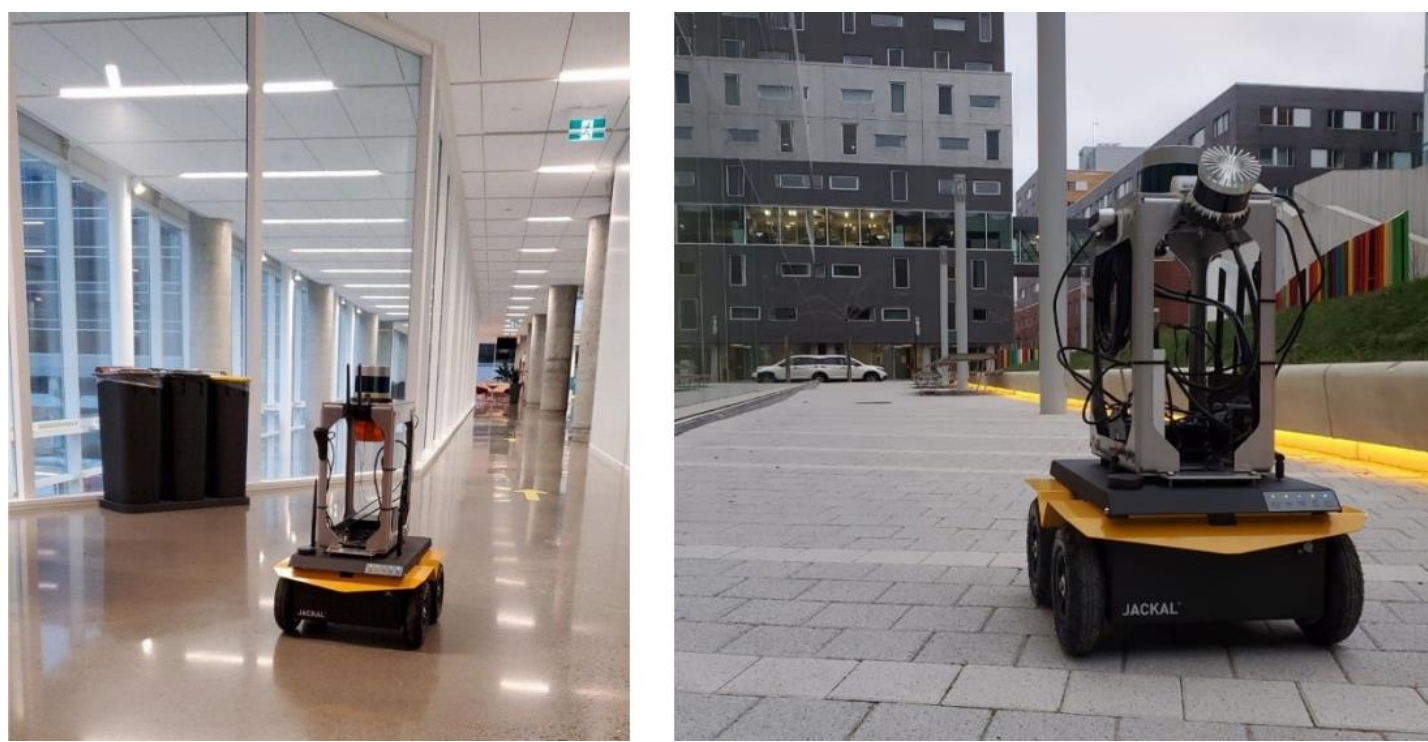

FIG. 9. Glass wall in "HALL 2044" (left) and outdoor environment (right).

Figure 9 illustrates that the glass wall is not detected by the navigation system (see left image of FIG. 8 and FIG. 9). Before entering the room, the navigation stack fetches information from the BIRS node about the next room. The material of that wall is listed as invisible to the sensor, so the navigation can rely on the BIM original layout instead of the robot's sensor thereby preventing the robot from colliding into the wall. Then, to pass through "VESTIBULE 2042", the robot's sensors are able to detect the door-way. However, the information of both doors for "VESTIBULE 2042" node such as door width, door height, and the central point of the door is also provided to complement the real-time sensor data. The robot's task is to enter room "W.C. HOMMES 2002" to scan the room and monitor if the equipment is installed. In the destination node (room) the robot calls the database to query which equipment is to be installed so that the data collected can be compared with the as-planned model.

\subsection{Semantic Outdoor Navigation}

This case study scenario relates to robot navigation in outdoor environments. In this use case, the robot navigates the site surrounding the building to collect data, while there are several obstacles to be detected through BIM-GIS, in order to contribute to obstacle avoidance. There is a landscape polygon covered with light vegetation, north of the building, located between Pavilion-D and another building on ÉTS campus. The polygon shape of the artifact contains 9 vertices. The coordinates of each vertex are retrieved from GIS and translated to the BIM environment. Similarly, the location of the vertices is provided by the BIRS node so that the navigation system establishes the polygon form as an obstacle. Furthermore, the outer boundary of the building is a wall, made of glass which the robot can hardly detect (see right image of FIG. 9). As shown in FIG. 10, all the information is encoded within an occupancy grid map compatible with ROS. The BIRS node provides the robot navigation system with all the required information, so it successfully complements the low-level information.

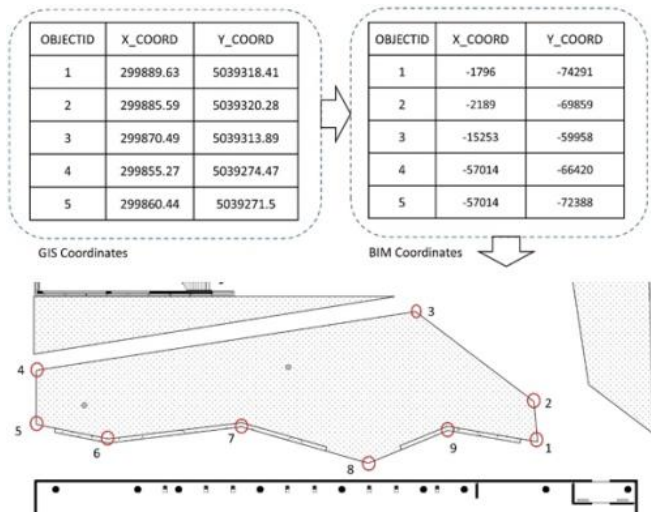

FIG. 10. Partial indication of coordinate transfer from GIS to BIM, which creates an occupancy grid map. 


\subsection{Progress Monitoring}

In the third scenario, the robot navigates the construction site and enters "CORRIDOR SUD 2010" (south hallway). As illustrated in FIG. 11, the robot generates a map (see left image of FIG. 11), which is the as-built map (real state) of the section covered by the robot. In this case, the as-planned map (see right part of FIG. 11) does not indicate wall at the time of data collection. This difference is not a sensors default; it shows that the project is ahead of schedule as the two walls on either side of the hallway were already installed. With the query script that was developed in the BIRS ontology, the robot has access to the BIM data and it fetches the relevant information. There are two uninstalled walls defined as landmarks as SubClassOf CORA:Region (SubClassOf SpatialStructureElement) in the BIRS ontology and their corresponding information is derived from IFC. As elaborated in the BIRS ontology, the wall on each side of "CORRIDOR SUD 2010" is a landmark; a building element defined by IfcBuildingElement. Corresponding information such as the GlobalID, location, material and room boundary relationships defined in the BIRS ontology is available through the BIRS node.
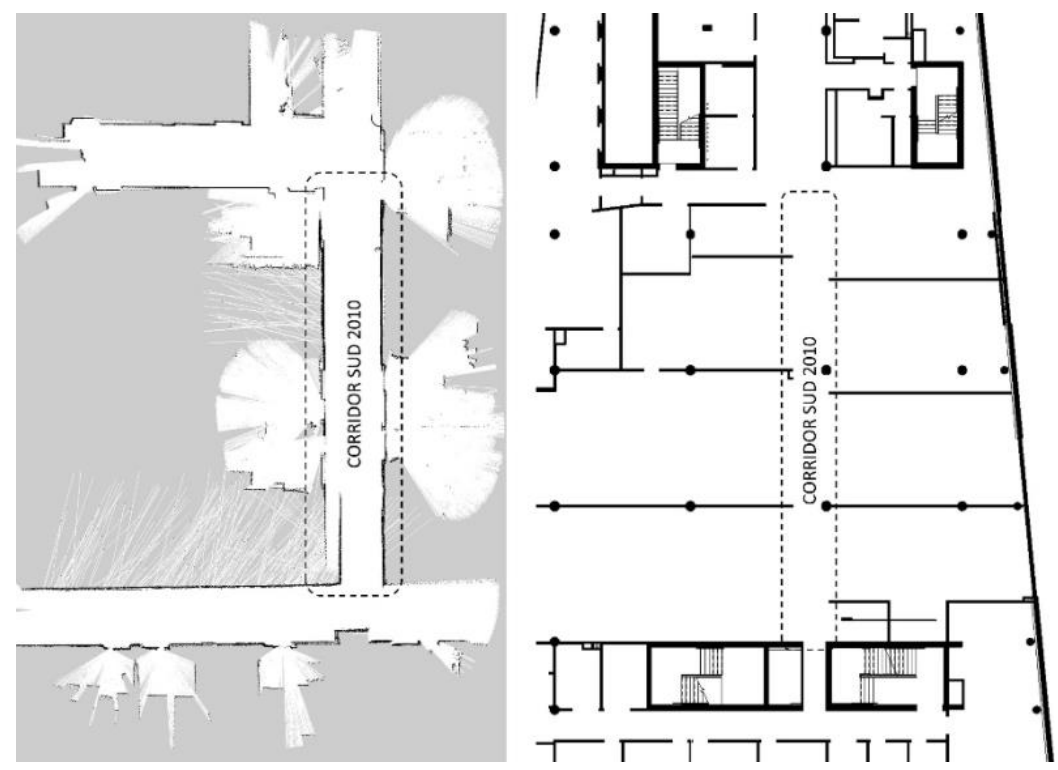

FIG. 11. The As-built map created by the robot (left) and As-planned map generated from BIM (right)

Based on the relevant information provided by the BIRS ontology, the robotic system is able to identify which walls are installed ahead of schedule. Similarly, this process can be used for all the building elements, which ultimately enables a detailed and accurate comparison between as-planned and as-built.

\subsection{Quality Control}

The last use case scenario illustrates quality control application of the BIRS ontology. In this scenario, when the robot is collecting data, it detects an irregularity, which is a state that requires assistance. While the robot is on its way from "CORRIDOR OUEST 2019" to "W.C. HOMMES 2002" to collect data - just before entering "VESTIBULE 2042" - the robot detects a column as a landmark that was not planned in that location. Figure 12 illustrates the as-planned building layout (see right image of FIG. 12) and the detected location of a column in the as-built map generated by the robot (see left image of FIG. 12). The BIRS ontology provides the information to the robot that no column is planned in that location. In this circumstance, an irregularity is detected on "NIVEAU 2 " and the robotic system finds the closest contractor's office to seek assistance. As illustrated in FIG. 12, the BIRS ontology provides the robot with the location of the contractor's office. The room's location is queried from the BIRS, so the robot can navigate to the office on its own.

The robot changes its path towards the contractor's office using a common warning signal to indicate that an irregularity was detected. This does not necessarily mean that the column was installed in an incorrect place. Still, it was not in the BIRS ontology and that is why the robot seeks assistance. This may happen when there is an obstacle in the occupancy grid map (generated by the robot) that is not in the map created from BIM. In this use case scenario, the detected irregularity is reported to the contractor for further analysis. A remote warning could also be sent if an operation room exists to supervise the robot's activities. 


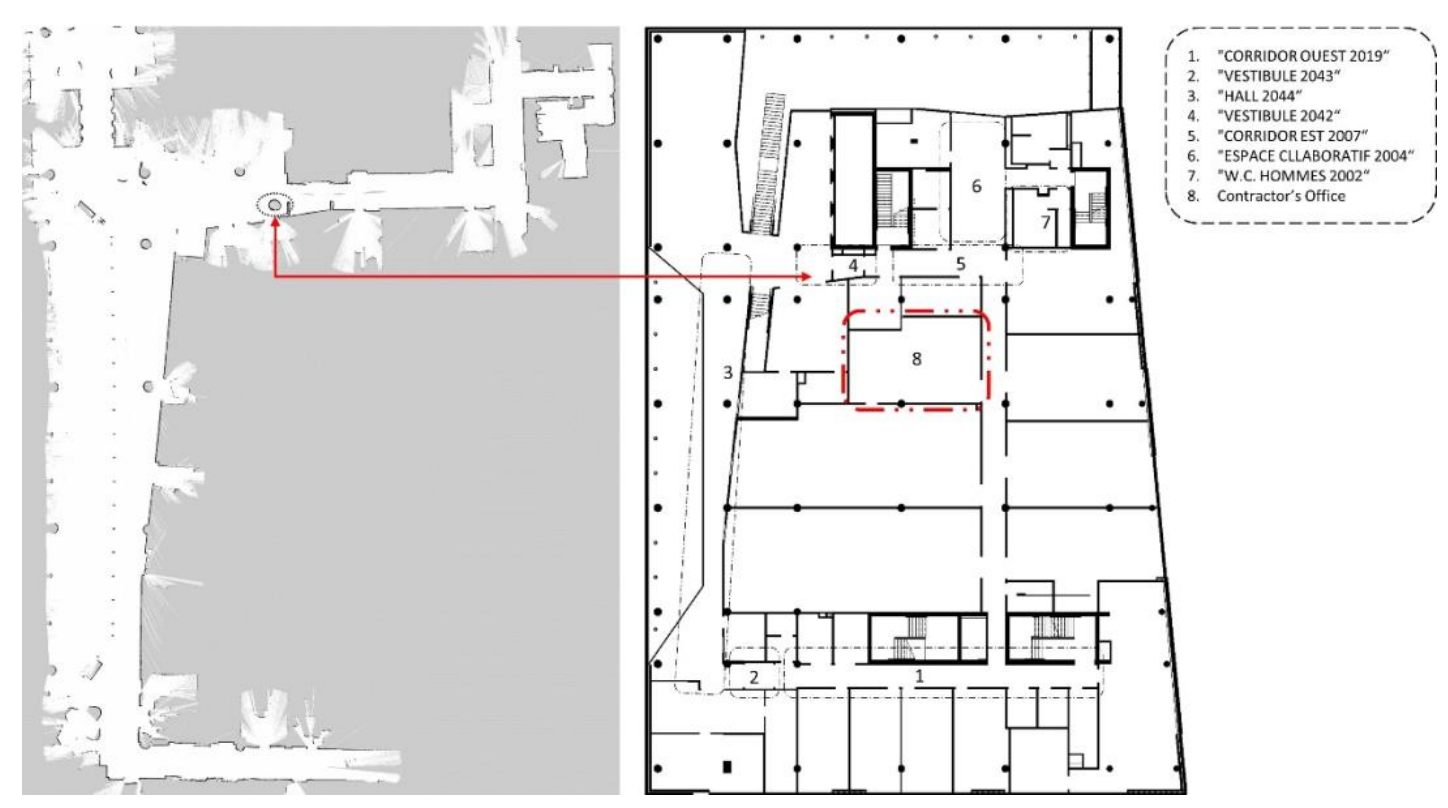

FIG. 12. The detected irregularity on the map by the robot (left) and the as-planned map generated from BIM (right)

\section{CONCLUSIONS}

The current study is part of a larger research project aiming to deploy automated data collection and progress monitoring of construction projects. Even if several studies address robot navigation, the problem of semantic data translation from BIM-GIS to the robot's navigation system was unsolved. Without a standard data format to translate data between BIM-GIS and ROS, building semantics cannot be leveraged by any robot navigation system. Therefore, this study presents semantic data exchanges between construction and robotics leveraging semantic web technology. To achieve this, an ontology-based approach is adopted bridging the knowledge representation of building-related data to two well-established ontologies, namely IEEE 1872-2015 and IEEE 1873-2015 standards; and this is the paper's first theoretical contribution. A BIRS ontology was developed to enable crossdomain data exchanges between both domains and, ultimately, the BIRS is implemented in four use cases on a construction site deploying autonomous robot navigation. The process is done by defining an intermediate level in the BIRS to bridge the knowledge representation. IFC and CityGML semantics are first stored in the topological database, parsed (using script developed in this study), and translated to ROS for the robot navigation stack to be understood and be utilized. A second type of data format leveraged is the occupancy grids common to the robotic domain that is generated from the construction data and is used in the full navigation system. Four use cases were conducted to test the application of the proposed ontology. The current paper's hypotheses were: (1) autonomous data collection using mobile robot can help in automated progress monitoring and (2) autonomous robot navigation can be improved by leveraging building-related data. The results show that the ontology provides semantic data that helps the robot to navigate and also understand what information is expected from its sensors, all of which can help to acquire accurate and more reliable data.

The BIRS applications can be extended to other construction areas such as calculating the productivity of the trades on site, creating an updated circulation map for mobile equipment, and creating evacuation plans for safety analysis. For example, the comparison between as-planned and as-built of a construction project can be made by comparing the map derived from BIM and the map generated by SLAM. Also, the information of Mechanical, Electrical, and Plumbing (MEP) equipment can be added to the ontology so that the data collected from the construction scene can be better compared to the as-built plan. In this sense, the robot can navigate the jobsite and collect data on a regular basis, thus providing information to the construction stakeholders as to the productivity of the construction project. While the robot is navigating the construction site, it collects data through its sensors and generates a map of the environment through SLAM. The SLAM-generated and BIM-driven maps complement each other and create an accurate map of the environment providing the location of the building elements and the temporary equipment and machinery on the site. The combined map can then be used to generate a circulation 
map for the construction site. In addition, this map can also be leveraged to create evacuation plans during the construction phase based on the existing conditions.

The current research takes IfcBuildingElement entity and its sub-classes into consideration to provide the robot with landmarks for navigation. However, the MEP equipment information is not included in the current study, i.e. if there is an equipment (obstacle for the robot), it would not appear on the map generated from BIM. This limitation can be addressed by extending the current ontology so that it contains MEP information in the BIRS ontology following the same methodology proposed in this paper. As shown in section 4.3, one of the use cases of the BIRS is progress monitoring. Including the MEP information of the building would provide more accurate and thorough progress monitoring of the construction project. The other limitation of this study is that the ontology aims to incorporate high-level semantic information of the building, while the practical robot navigation system requires low-level information provided by sensors and the strategies to implement low-level navigation. The topological map developed in this paper is a rich database of building elements semantics that can be used for semantic path planning for robot navigation. As robot navigation is highly dependent on low-level sensor-based navigation, the authors aim to develop a strategy that uses the topological map and sensors information to implement a full-stack semantic solution to enable mobile robots to navigate on construction sites.

\section{ACKNOWLEDGEMENT}

The authors are grateful to the Natural Sciences and Engineering Research Council of Canada for its financial support through CRD program 543867-2019 as well as to Pomerleau - the industrial partner of the ÉTS Industrial Chair on the Integration of Digital Technology in Construction.

\section{REFERENCES}

Afkhamiaghda M., Mahdaviparsa A., Afsari K. and McCuen T. (2019). Occupants behavior-based design study using BIM-GIS integration: An alternative design approach for architects, Advances in Informatics and Computing in Civil and Construction Engineering. Springer, pp. 765-772.

Afsari K., Florez L., Maneke E. and Afkhamiaghda M. (2019). An Experimental Investigation of the Integration of Smart Building Components with Building Information Model (BIM), Proceedings of the 36th International Symposium on Automation and Robotics in Construction (ISARC). International Association for Automation and Robotics in Construction (IAARC), pp. 578-585.

Albeaino G. and Gheisari M. (2021). Trends, benefits, and barriers of unmanned aerial systems in the construction industry: a survey study in the United States. Journal of Information Technology in Construction (ITcon) 26, 84-111.

Albeaino G., Gheisari M. and Franz B.W. (2019). A systematic review of unmanned aerial vehicle application areas and technologies in the AEC domain. ITcon 24, 381-405.

Almasri M., Elleithy K. and Alajlan A. (2016). Sensor fusion based model for collision free mobile robot navigation. Sensors 16, 24.

Ardiny H., Witwicki S. and Mondada F. (2015). Construction Automation with Autonomous Mobile Robots: A Review. pp. 418-424. https://doi.org/10.1109/ICRoM.2015.7367821

Asadi K., Kalkunte Suresh A., Ender A., Gotad S., Maniyar S., Anand S., Noghabaei M., Han K., Lobaton E. and Wu T. (2020). An integrated UGV-UAV system for construction site data collection. Automation in Construction 112, 103068. https://doi.org/10.1016/j.autcon.2019.103068

Atazadeh B., Kalantari M., Rajabifard A., Ho S. and Ngo T. (2017). Building information modelling for high-rise land administration. Transactions in GIS 21, 91-113.

Aziz Z., Anumba C.J., Ruikar D., Carrillo P. and Bouchlaghem D. (2006). Intelligent wireless web services for construction-A review of the enabling technologies. Automation in Construction 15, 113-123.

Barbau R., Krima S., Rachuri S., Narayanan A., Fiorentini X., Foufou S. and Sriram R.D. (2012). OntoSTEP: Enriching product model data using ontologies. Computer-Aided Design 44, 575-590. 
Barbosa F., Woetzel J., Mischke J., Ribeirinho M.J., Sridhar M., Parsons M., Bertram N. and Brown S. (2017). Reinventing construction: A route to higher productivity. McKinsey Global Institute.

Beetz M., Beßler D., Haidu A., Pomarlan M., Bozcuoğlu A.K. and Bartels G. (2018). Know rob 2.0—a 2nd generation knowledge processing framework for cognition-enabled robotic agents, in: 2018 IEEE International Conference on Robotics and Automation (ICRA). IEEE, pp. 512-519.

Beetz M., Tenorth M. and Winkler J. (2015). Open-ease, 2015 IEEE International Conference on Robotics and Automation (ICRA). IEEE, pp. 1983-1990.

Berners-Lee T. and Hendler J. (2001). Publishing on the semantic web. Nature 410, 1023-1024.

buildingSMART, 2013. $\quad$ IfcSpace. $\quad$ IfcSpace. https://standards.buildingsmart.org/IFC/RELEASE/IFC2x3/FINAL/HTML/ifcproductextension/lexical/if cspace.htm (accessed 1.27.21).

Chbeir R., Cardinale Y., Corchero A., Bourreau P., Salameh K., Charbel N., Kallab L., Angsuchotmetee C. and Calis G. (2018). OntoH2G: a semantic model to represent building infrastructure and occupant interactions, International Conference on Sustainability in Energy and Buildings. Springer, pp. 148-158.

Choi J., Choi M., Nam S.Y. and Chung W.K. (2011). Autonomous topological modeling of a home environment and topological localization using a sonar grid map. Autonomous Robots 30, 351-368.

Crespo J., Castillo J.C., Mozos O.M. and Barber R. (2020). Semantic Information for Robot Navigation: A Survey. Applied Sciences 10, 497.

Delbrügger T., Lenz L.T., Losch D. and Roßmann J. (2017). A navigation framework for digital twins of factories based on building information modeling, 2017 22nd IEEE International Conference on Emerging Technologies and Factory Automation (ETFA). IEEE, pp. 1-4.

Diab M., Akbari A., Ud Din M., Rosell J. (2019). Pmk-a knowledge processing framework for autonomous robotics perception and manipulation. Sensors 19, 1166.

Doumbouya L., Guan C.S., Gao G. and Pan Y. (2017). Application of BIM technology in design and construction: A case study of pharmaceutical industrial base of amino acid building project. Engineering for Rural Development 16, 1495-02.

El-Diraby T.A., Lima C. and Feis, B. (2005). Domain taxonomy for construction concepts: toward a formal ontology for construction knowledge. Journal of computing in civil engineering 19, 394-406.

Elghamrawy T. and Boukamp F. (2010). Managing construction information using RFID-based semantic contexts. Automation in construction 19, 1056-1066.

Elghamrawy T. and Boukamp F. (2008). A vision for a framework to support management of and learning from construction problems, Proceedings of the 25th International Conference on Formation Technology in Construction: Improving the Management of Construction Projects Through IT Adoption, Santiago, Chile.

Esan O., Du S. and Lodewyk B. (2020). Review on Autonomous Indoor Wheel Mobile Robot Navigation Systems, in: 2020 International Conference on Artificial Intelligence, Big Data, Computing and Data Communication Systems (IcABCD). IEEE, pp. 1-6.

Farias M.T., Roxin A. and Nicolle C. (2015). Cobieowl, an owl ontology based on cobie standard, OTM Confederated International Conferences" On the Move to Meaningful Internet Systems". Springer, pp. 361-377.

Fiorini S.R., Carbonera J.L., Gonçalves P., Jorge V.A.M., Rey V.F., Haidegger T., Abel M., Redfield S.A., Balakirsky S., Ragavan V., Li H., Schlenoff C. and Prestes E. (2015). Extensions to the core ontology for robotics and automation. Robotics and Computer-Integrated Manufacturing 33, 3-11. https://doi.org/10.1016/j.rcim.2014.08.004 
Galindo C., Saffiotti A., Coradeschi S., Buschka P., Fernandez-Madrigal J.-A. and González J. (2005). Multihierarchical semantic maps for mobile robotics, 2005 IEEE/RSJ International Conference on Intelligent Robots and Systems. IEEE, pp. 2278-2283.

González E., Piñeiro J.D., Toledo J., Arnay R. and Acosta L. (2020). An approach based on the ifcOWL ontology to support indoor navigation. Egyptian Informatics Journal.

Gruber T.R. (1995). Toward principles for the design of ontologies used for knowledge sharing? International journal of human-computer studies 43, 907-928.

Guarino N. (1995). Formal ontology, conceptual analysis and knowledge representation. International journal of human-computer studies 43, 625-640.

Hamieh A., Deneux D. and Tahon C. (2017). BiMov: BIM-Based Indoor Path Planning, (Eynard, B., Nigrelli, V., Oliveri, S.M., Peris-Fajarnes, G., Rizzuti, S., editors), Advances on Mechanics, Design Engineering and Manufacturing: Proceedings of the International Joint Conference on Mechanics, Design Engineering \& Advanced Manufacturing (JCM 2016), 14-16 September, 2016, Catania, Italy. Springer International Publishing, Cham, pp. 889-899. https://doi.org/10.1007/978-3-319-45781-9_89

Hassan A.M., Elias C.M., Shehata O.M. and Morgan E.I. (2017). A global integrated artificial potential field/virtual obstacles path planning algorithm for multi-robot system applications. Int. Research J. of Eng. and Technology 4, 1198-1204.

Hatori F., Satou K., Onodera J. and Yashiro Y. (2020). Development of BIM-Based 4D Simulation System for Construction Schedule Planning, International Conference on Computing in Civil and Building Engineering. Springer, pp. 547-560.

Ibrahim A., Roberts D., Golparvar-Fard M. and Bretl T. (2017). An Interactive Model-Driven Path Planning and Data Capture System for Camera-Equipped Aerial Robots on Construction Sites, Computing in Civil Engineering 2017. pp. 117-124.

IEEE Standard for Robot Map Data Representation for Navigation. (2015). 1873-2015 IEEE Standard for Robot Map Data Representation for Navigation 1-54. https://doi.org/10.1109/IEEESTD.2015.7300355

IEEE Standard Ontologies for Robotics and Automation. (2015). IEEE Std 1872-2015 1-60. https://doi.org/10.1109/IEEESTD.2015.7084073

Irizary J. and Karan E. (2012). Optimizing location of tower cranes on construction sites through GIS and BIM integration. Electronic Journal of Information Technology in Construction 17, 351-366.

Isikdag U., Zlatanova S. and Underwood J. (2013). A BIM-Oriented Model for supporting indoor navigation requirements. Computers, Environment and Urban Systems 41, 112-123. https://doi.org/10.1016/j.compenvurbsys.2013.05.001

Kalfoglou Y. (2009). Cases on Semantic Interoperability for Information Systems Integration: Practices and Applications: Practices and Applications. IGI Global.

Karan E. P., Irizarry J. and Haymaker J. (2016). BIM and GIS Integration and Interoperability Based on Semantic Web Technology. Journal of Computing in Civil Engineering 30, 04015043. https://doi.org/10.1061/(ASCE)CP.1943-5487.0000519

Karan E. P., Sivakumar R., Irizarry J. and Guhathakurta S. (2014). Digital Modeling of Construction Site Terrain Using Remotely Sensed Data and Geographic Information Systems Analyses. Journal of Construction Engineering and Management 140, 04013067. https://doi.org/10.1061/(ASCE)CO.1943-7862.0000822

Karan E.P. and Irizarry J. (2015). Extending BIM interoperability to preconstruction operations using geospatial analyses and semantic web services. Automation in Construction 53, 1-12. https://doi.org/10.1016/j.autcon.2015.02.012 
Karimi S. and Iordanova I. (2021). Integration of BIM and GIS for Construction Automation, a Systematic Literature Review (SLR) Combining Bibliometric and Qualitative Analysis. Archives of Computational Methods in Engineering 1-22.

Kollar T. and Roy N. (2009). Utilizing object-object and object-scene context when planning to find things, in: 2009 IEEE International Conference on Robotics and Automation. IEEE, pp. 2168-2173.

Kostavelis I. and Gasteratos A. (2017). Semantic maps from multiple visual cues. Expert Systems with Applications 68, 45-57.

Lin J.J. and Golparvar-Fard M. (2020). Construction Progress Monitoring Using Cyber-Physical Systems, in: Anumba, C.J., Roofigari-Esfahan, N. (Eds.), Cyber-Physical Systems in the Built Environment. Springer International Publishing, Cham, pp. 63-87. https://doi.org/10.1007/978-3-030-41560-0_5

Liu X., Li Z. and Jiang S. (2016). Ontology-based representation and reasoning in building construction cost estimation in China. Future Internet 8, 39.

Niloy A.K., Shama A., Chakrabortty R.K., Ryan M.J., Badal F.R., Tasneem Z., Ahamed M.H., Moyeen S.I., Das S.K., Ali M.F. and Islam M.R. (2021). Critical Design and Control Issues of Indoor Autonomous Mobile Robots: A Review. IEEE Access.

Meschini S., Iturralde K., Linner T. and Bock T. (2016). Novel applications offered by integration of robotic tools in BIM-based design workflow for automation in construction processes, Proceedings of the CIB* IAARC W119 CIC 2016 Workshop.

Mignard C. and Nicolle C. (2014). Merging BIM and GIS using ontologies application to urban facility management in ACTIVe3D. Computers in Industry 65, 1276-1290. https://doi.org/10.1016/j.compind.2014.07.008

Mirats-Tur J.M., Zinggerling C. and Corominas-Murtra A. (2009). GIS map based mobile robot navigation in urban environments, in: 2009 International Conference on Advanced Robotics. IEEE, pp. 1-6.

Nahangi M., Heins A., McCabe B. and Schoellig A., (2018). Automated localization of UAVs in GPS-denied indoor construction environments using fiducial markers, ISARC - Int. Symp. Autom. Robot. in Constr. Int. AEC/FM Hackathon: The Future of Build. Things. Presented at the 35th International Symposium on Automation and Robotics in Construction and International AEC/FM Hackathon: The Future of Building Things, ISARC 2018, International Association for Automation and Robotics in Construction I.A.A.R.C).

Niles I. and Pease A. (2001). Towards a standard upper ontology, Proceedings of the International Conference on Formal Ontology in Information Systems-Volume 2001. pp. 2-9.

Olivares-Alarcos A., Beßler D., Khamis A., Goncalves P., Habib M.K., Bermejo-Alonso J., Barreto M., Diab M., Rosell J. and Quintas, J. (2019). A review and comparison of ontology-based approaches to robot autonomy. The Knowledge Engineering Review 34.

Palacz W., Ślusarczyk G., Strug B. and Grabska E. (2019). Indoor Robot Navigation Using Graph Models Based on BIM/IFC, in: Rutkowski, L., Scherer, R., Korytkowski, M., Pedrycz, W., Tadeusiewicz, R., Zurada, J.M. (Eds.), Artificial Intelligence and Soft Computing. Springer International Publishing, Cham, pp. 654665.

Pauwels P., Terkaj W., Krijnen T. and Beetz J. (2015). Coping with lists in the ifcOWL ontology, in: 22nd EGICE International Workshop. pp. 113-122.

Pauwels P., Zhang S. and Lee Y.-C. (2017). Semantic web technologies in AEC industry: A literature overview. Automation in Construction 73, 145-165. https://doi.org/10.1016/j.autcon.2016.10.003

Persson J., Gallois A., Björkelund A., Hafdell L., Haage M., Malec J., Nilsson K. and Nugues P. (2010). A knowledge integration framework for robotics, in: ISR 2010 (41st International Symposium on Robotics) and ROBOTIK 2010 (6th German Conference on Robotics). VDE, pp. 1-8. 
Prestes E., Carbonera J.L., Fiorini S.R., Jorge V.A., Abel M., Madhavan R., Locoro A., Goncalves P., Barreto M.E. and Habib M. (2013). Towards a core ontology for robotics and automation. Robotics and Autonomous Systems 61, 1193-1204.

Yang Q., Wang M., Kwan M.P. and Yang Y. (2015). A novel GIS platform for UGV application in the unknown environment, in: 2015 23rd International Conference on Geoinformatics. Presented at the 2015 23rd International Conference on Geoinformatics, $\quad$ pp. https://doi.org/10.1109/GEOINFORMATICS.2015.7378630

Rebolj D., Pučko Z., Babič N.Č., Bizjak M. and Mongus D. (2017). Point cloud quality requirements for Scan-vsBIM based automated construction progress monitoring. Automation in Construction 84, 323-334. https://doi.org/10.1016/j.autcon.2017.09.021

Khruahong S., Kong X., Sandrasegaran K. and Liu L. (2017). Multi-level indoor navigation ontology for high assurance location-based services. In 2017 IEEE 18th International Symposium on High Assurance Systems Engineering (HASE) (pp. 128-131). IEEE.

Savitri D.M. and Pramudya A.A. (2020). Clash detection analysis with BIM-based software on midrise building construction project. IOP Conference Series: Earth and Environmental Science (Vol. 426, No. 1, p. 012002). IOP Publishing.

Siemiątkowska B., Harasymowicz-Boggio B., Przybylski M., Różańska-Walczuk M., Wiśniowski M. and Kowalski M. (2013). BIM based indoor navigation system of Hermes mobile robot, Romansy 19-Robot Design, Dynamics and Control. Springer, pp. 375-382.

Staub-French S. and Nepal M.P. (2007). Reasoning about component similarity in building product models from the construction perspective. Automation in Construction 17, 11-21.

Streule T., Miserini N., Bartlomé O., Klippel M. and de Soto B.G. (2016). Implementation of Scrum in the Construction Industry. Procedia Engineering 164, 269-276. https://doi.org/10.1016/j.proeng.2016.11.619

Studer R., Benjamins V.R. and Fensel D. (1998). Knowledge engineering: Principles and methods. Data \& knowledge engineering 25, 161-197.

Tashakkori H., Rajabifard A. and Kalantari M. (2015). A new 3D indoor/outdoor spatial model for indoor emergency response facilitation. Building and Environment 89, 170-182. https://doi.org/10.1016/j.buildenv.2015.02.036

Tenorth M. and Beetz M. (2009). KnowRob—-knowledge processing for autonomous personal robots, in: 2009 IEEE/RSJ International Conference on Intelligent Robots and Systems. IEEE, pp. 4261-4266.

Teo T.-A. and Cho K.-H. (2016). BIM-oriented indoor network model for indoor and outdoor combined route planning. Advanced Engineering Informatics 30, 268-282. https://doi.org/10.1016/j.aei.2016.04.007

Terkaj W. and Viganò G.P. (2017). Semantic GIOVE-VF: An Ontology-Based Virtual Factory Tool., in: JOWO.

Usmani A.U., Jadidi M. and Sohn G. (2020). Automatic Ontology Generation of Bim and GIS Data. The International Archives of Photogrammetry, Remote Sensing and Spatial Information Sciences 43, 77-80.

Van Rees R. (2003). Clarity in the usage of the terms ontology, taxonomy and classification. CIB REPORT 284, $1-8$.

Venugopal M., Eastman C.M. and Teizer J. (2015). An ontology-based analysis of the industry foundation class schema for building information model exchanges. Advanced Engineering Informatics 29, 940-957. https://doi.org/10.1016/j.aei.2015.09.006

Venugopal M., Eastman C.M. and Teizer J. (2012). An ontological approach to building information model exchanges in the precast/pre-stressed concrete industry, Construction Research Congress 2012: Construction Challenges in a Flat World. pp. 1114-1123. 
Wang H., Pan Y. and Luo X. (2019). Integration of BIM and GIS in sustainable built environment: A review and bibliometric analysis. Automation in Construction 103, 41-52. https://doi.org/10.1016/j.autcon.2019.03.005

Wang N. and Issa R.R. (2020). Ontology-Based Integration of BIM and GIS for Indoor Routing, in: Construction Research Congress 2020: Computer Applications. American Society of Civil Engineers Reston, VA, pp. 1010-1019.

Wang X., Shi Y., Ding D. and Gu X. (2016). Double global optimum genetic algorithm-particle swarm optimization-based welding robot path planning. Engineering Optimization 48, 299-316.

World Wide Web Consortium. (2014). Resource Description Framework (RDF). URL https://www.w3.org/RDF/ (accessed 1.18.21)

Yamamura S., Fan L. and Suzuki Y. (2017). Assessment of Urban Energy Performance through Integration of BIM and GIS for Smart City Planning. Procedia Engineering 180, 1462-1472. https://doi.org/10.1016/j.proeng.2017.04.309

Yan D.H., Zhao D.A. and Shen H.L. (2013). The mobile robot navigation system design based on GPS and GIS, in: Applied Mechanics and Materials. Trans Tech Publ, pp. 1918-1921.

Zhu J., Wright G., Wang J. and Wang X. (2018). A critical review of the integration of geographic information system and building information modelling at the data level. ISPRS International Journal of GeoInformation 7, 66. 